\title{
Running Coupling Evolution for Diffractive Dissociation and the NLO Odderon Intercept
}

\author{
Yuri V. Kovchegov \\ Department of Physics, The Ohio State University, Columbus, OH 43210, USA
}

\begin{abstract}
We summarize the results of including running coupling corrections into the nonlinear evolution equation for diffractive dissociation. We also document a prediction that the NLO QCD odderon intercept is zero resulting from a discussion at the Diffraction 2012 Workshop.
\end{abstract}

Keywords: Diffraction, Nonlinear Evolution, Odderon

PACS: 12.38.-t, 12.38.Bx, 12.38.Cy

\section{RUNNING COUPLING CORRECTIONS FOR DIFFRACTIVE DISSOCIATION}

This proceedings contribution is mainly based on the paper [1].

The evolution equation for single diffractive dissociation in deep inelastic scattering (DIS) was derived in [2]. First we write the cross section for single diffractive dissociation in DIS on a nucleus as

$$
M_{X}^{2} \frac{d \sigma_{d i f f}^{\gamma^{*} A}}{d M_{X}^{2}}=-\int d^{2} x_{0} d^{2} x_{1} \int_{0}^{1} d z\left|\Psi^{\gamma^{*} \rightarrow q \bar{q}}\left(x_{01}, z\right)\right|^{2} \frac{\partial S_{\mathbf{x}_{0}, \mathbf{x}_{1}}^{D}\left(Y, Y_{0}\right)}{\partial Y_{0}},
$$

where $Y=\ln \left(s / Q^{2}\right)$ is the net rapidity interval and the rapidity gap stretches from rapidity 0 to rapidity $Y_{0} \approx \ln \left(s / M_{X}^{2}\right)$ with $M_{X}^{2}$ the invariant mass of the produced hadrons. $\left|\Psi^{\gamma^{*} \rightarrow q \bar{q}}\left(x_{01}, z\right)\right|^{2}$ is the order- $\alpha_{E M}$ light-cone wave function squared for a virtual photon fluctuating into a $q \bar{q}$ pair with $x_{01}=\left|\mathbf{x}_{0}-\mathbf{x}_{1}\right|$ the transverse size of the pair and $z$ the fraction of the longitudinal momentum of the incoming virtual photon carried by the quark in the pair. The object $S^{D}$ is the "S-matrix" for single diffractive dissociation, which includes both interacting and non-interacting contributions in the amplitude and in the complex conjugate amplitude with the rapidity gap greater than or equal to $Y_{0}[1]$.

The object $S^{D}$ obeys a nonlinear evolution equation, which is equivalent to the Balitsky-Kovchegov (BK) [3, 4] evolution equation [1, 2, 5]:

$$
\partial_{Y} S_{\mathbf{x}_{0}, \mathbf{x}_{1}}^{D}\left(Y, Y_{0}\right)=\frac{\alpha_{S} N_{c}}{2 \pi^{2}} \int d^{2} x_{2} \frac{x_{10}^{2}}{x_{20}^{2} x_{21}^{2}}\left[S_{\mathbf{x}_{0}, \mathbf{x}_{2}}^{D}\left(Y, Y_{0}\right) S_{\mathbf{x}_{2}, \mathbf{x}_{1}}^{D}\left(Y, Y_{0}\right)-S_{\mathbf{x}_{0}, \mathbf{x}_{1}}^{D}\left(Y, Y_{0}\right)\right]
$$

The equation is illustrated in Fig. 1. The solid vertical line in Fig. 1 denotes the finalstate cut, while the vertical dashed lines denote interactions with the target nucleus in the amplitude and in the complex-conjugate amplitude. The initial condition for the 


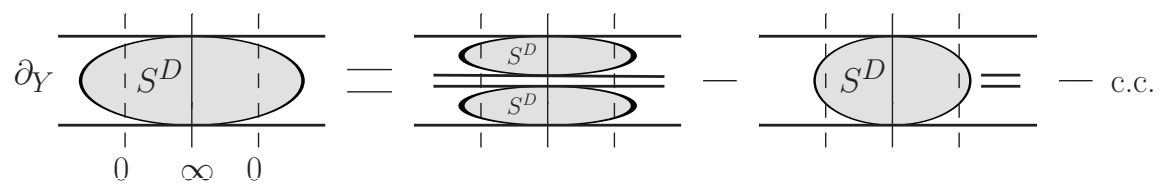

FIGURE 1. The evolution equation for $S^{D}$.

evolution (2) is given by

$$
S_{\mathbf{x}_{0}, \mathbf{x}_{1}}^{D}\left(Y=Y_{0}, Y_{0}\right)=\left[1-N_{\mathbf{x}_{0}, \mathbf{x}_{1}}\left(Y_{0}\right)\right]^{2},
$$

where $N$ is the (imaginary part) of the forward dipole-nucleus scattering amplitude obeying the standard BK evolution equation.

It can be shown [1] that the fact that $S^{D}$ obeys the BK evolution results from the cancellation of final state gluon emissions: no $s$-channel gluon emitted or absorbed after the interaction with the target (to the right of the dashed line in the amplitude and to the left of the dashed line in the complex conjugate amplitude) remains in the final evolution pictured in Fig. 1. Without the final state gluon emissions/absorptions, the evolution becomes just like that for a forward amplitude, that is, a BK evolution. The same property remains valid when the running coupling corrections are included [1]: these corrections also cancel in the final state. Hence, the running-coupling evolution equation for diffractive dissociation is equivalent to the running-coupling BK ( $\mathrm{rcBK}$ ) evolution equation, with the initial condition (3) now containing the dipole amplitude $N$ evolved by the full rcBK evolution. We thus write the running-coupling evolution equation for $S^{D}$ as [1]

$$
\partial_{Y} S_{\mathbf{x}_{0}, \mathbf{x}_{1}}^{D}\left(Y, Y_{0}\right)=\int d^{2} x_{2} K\left(\mathbf{x}_{0}, \mathbf{x}_{1}, \mathbf{x}_{2}\right)\left[S_{\mathbf{x}_{0}, \mathbf{x}_{2}}^{D}\left(Y, Y_{0}\right) S_{\mathbf{x}_{2}, \mathbf{x}_{1}}^{D}\left(Y, Y_{0}\right)-S_{\mathbf{x}_{0}, \mathbf{x}_{1}}^{D}\left(Y, Y_{0}\right)\right]
$$

where the evolution kernel was calculated in [6,7]. For completeness let us show the kernel in the Balitsky prescription [7]:

$$
K_{r c}^{B a l}\left(\mathbf{x}_{0}, \mathbf{x}_{1}, \mathbf{x}_{2}\right)=\frac{N_{c} \alpha_{s}\left(x_{10}^{2}\right)}{2 \pi^{2}}\left[\frac{x_{10}^{2}}{x_{20}^{2} x_{21}^{2}}+\frac{1}{x_{20}^{2}}\left(\frac{\alpha_{s}\left(x_{20}^{2}\right)}{\alpha_{s}\left(x_{21}^{2}\right)}-1\right)+\frac{1}{x_{21}^{2}}\left(\frac{\alpha_{s}\left(x_{21}^{2}\right)}{\alpha_{s}\left(x_{20}^{2}\right)}-1\right)\right],
$$

where $\alpha_{S}\left(x_{\perp}^{2}\right)=\alpha_{S}\left(4 e^{-\frac{5}{3}-2 \gamma_{E}} / x_{\perp}^{2}\right)$.

Eq. (4) can be used to describe the DIS diffraction data with large center-of-mass energy squared $s$ and large $M_{X}^{2}$, such that $s \gg M_{X}^{2} \gg Q^{2}$. Unfortunately current HERA data does not extend to high enough values of $M_{X}^{2}$ to necessitate the use of Eq. (4): perhaps this equation would be useful to describe single diffraction at the future DIS machines such as the proposed EIC and LHeC colliders.

\section{THE NLO ODDERON INTERCEPT}

The progress in the calculation of the next-to-leading order (NLO) intercept of the QCD odderon was presented in the talk by Jochen Bartels at the Diffraction 2012 
Workshop (reporting on work being done in collaboration with Victor Fadin and Lev Lipatov). The calculation employs standard Feynman diagram approach. Here we would like to document a prediction for the NLO odderon intercept made by the author of these proceedings in the discussion following the talk: the NLO odderon intercept can be straightforwardly obtained using the $s$-channel time-ordered formalism usually employed in saturation physics.

The odderon exchange amplitude in DIS is [8]

$$
O_{\mathbf{x y}}=\frac{1}{2 i} \frac{1}{N_{c}}\left\langle\operatorname{Tr}\left[V_{\mathbf{x}} V_{\mathbf{y}}^{\dagger}\right]-\operatorname{Tr}\left[V_{\mathbf{y}} V_{\mathbf{x}}^{\dagger}\right]\right\rangle=\frac{1}{2 i}\left[N_{\mathbf{y}, \mathbf{x}}-N_{\mathbf{x}, \mathbf{y}}\right]
$$

where $V_{\mathbf{x}}$ is a Wilson line along the light-cone of the projectile dipole located at transverse coordinate $\mathbf{x}$ and $N_{\mathbf{x}, \mathbf{y}}=1-\left\langle\operatorname{Tr}\left[V_{\mathbf{x}} V_{\mathbf{y}}^{\dagger}\right] / N_{c}\right\rangle$ is the dipole-nucleus forward scattering amplitude.

To construct the NLO evolution equation for $O_{\mathbf{x y}}$ we begin with the linearized NLO BK evolution for $N_{\mathbf{x}, \mathbf{y}}$ derived in [9] (that is, the NLO Balitsky-Fadin-Kuraev-Lipatov (BFKL) equation $[10,11]$ in transverse coordinate space), which can be written as (see Eq. (103) in [9])

$$
\partial_{Y} N_{\mathbf{x}, \mathbf{y}}=\int d^{2} z K_{1}(\mathbf{x}, \mathbf{y} ; \mathbf{z})\left[N_{\mathbf{x}, \mathbf{z}}+N_{\mathbf{z}, \mathbf{y}}-N_{\mathbf{x}, \mathbf{y}}\right]+\int d^{2} z d^{2} z^{\prime} K_{2}\left(\mathbf{x}, \mathbf{y} ; \mathbf{z}, \mathbf{z}^{\prime}\right) N_{\mathbf{z}, \mathbf{z}^{\prime}}
$$

The kernels $K_{1}$ and $K_{2}$ can be found in Eq. (103) of [9]. One can explicitly verify that

$$
K_{1}(\mathbf{x}, \mathbf{y} ; \mathbf{z})=K_{1}(\mathbf{y}, \mathbf{x} ; \mathbf{z}) \text { and } K_{2}\left(\mathbf{x}, \mathbf{y} ; \mathbf{z}, \mathbf{z}^{\prime}\right)=K_{2}\left(\mathbf{y}, \mathbf{x} ; \mathbf{z}^{\prime}, \mathbf{z}\right)
$$

Using these properties of the kernels, along with Eqs. (7) and (6), we derive the NLO evolution equation for the odderon amplitude, which turns out to be equivalent to Eq. (7):

$$
\partial_{Y} O_{\mathbf{x}, \mathbf{y}}=\int d^{2} z K_{1}(\mathbf{x}, \mathbf{y} ; \mathbf{z})\left[O_{\mathbf{x}, \mathbf{z}}+O_{\mathbf{z}, \mathbf{y}}-O_{\mathbf{x}, \mathbf{y}}\right]+\int d^{2} z d^{2} z^{\prime} K_{2}\left(\mathbf{x}, \mathbf{y} ; \mathbf{z}, \mathbf{z}^{\prime}\right) O_{\mathbf{z}, \mathbf{z}^{\prime}}
$$

We see that the situation closely replicates that for the odderon evolution in transverse coordinate space at the leading order (LO) [12]: there the odderon evolution equation was also identical to the (LO) BFKL equation, with the eigenfunctions of the odderon evolution operator being $C$-odd, that is, they had to flip sign under the $\mathbf{x} \leftrightarrow \mathbf{y}$ interchange. The same observation applies here at NLO: since the odderon amplitude $O$ obeys the same NLO BFKL evolution equation, the odderon intercept is the same as the NLO BFKL intercept, with only odd values of the azimuthal index $n$ contributing. (Note that, strictly speaking, to obtain Eq. (7) which is equivalent to NLO BFKL one has to redefine the dipole amplitude by an order- $\alpha_{s}$ correction with the corresponding new operator referred to as the composite dipole in [13]: using this operator in place of $N$ would not change the derivation above.)

The NLO BFKL intercept for non-zero $n$ can be found in e.g. [14] (see Eq. (65) there). Evaluating it for $n=1$ at the saddle point $\gamma=\frac{1}{2}+i v=\frac{1}{2}$ we obtain zero ${ }^{1}$, such that the

\footnotetext{
1 The fact that the NLO BFKL intercept is zero for $n=1$ and $\gamma=1 / 2$ had been originally observed by Agustin Sabio Vera during the discussion of the odderon evolution at the Diffraction 2012 Workshop (see also Eq. (38) in [15]), and was since confirmed by the author.
} 
NLO odderon intercept in QCD is

$$
\alpha_{O}-1=0+O\left(\alpha_{s}^{3}\right) .
$$

We conclude that the zero value of the odderon intercept, originally found at the leadingorder in [16], persists at the NLO. ${ }^{2}$

It is interesting to note that, as was pointed out by Lipatov in the discussion at the Workshop, the odderon intercept at strong 't Hooft coupling $\lambda=g^{2} N_{c}$ was found in [18] using the Anti-de Sitter space/Conformal Field Theory (AdS/CFT) correspondence. The authors of [18] found two odderon solutions, both of which give $\alpha_{O}-1 \rightarrow 0$ for $\lambda \rightarrow \infty$, with one of the solutions exhibiting no deviation from zero when finite- $\lambda$ corrections were calculated, such that $\alpha_{O}-1=0+O(1 / \lambda)$. This result, along with our Eq. (10), presents evidence in favor of a tantalizing possibility that the odderon intercept is identically equal to zero at all values of the coupling! (The same reference [18] states that the result (10) had been known earlier to Cyrille Marquet, who, as it turns out, arrived at it numerically using a similar line of arguments to the one presented here.)

\section{ACKNOWLEDGMENTS}

The author is greatly indebted to Ian Balitsky and Giovanni Chirilli for discussions of the NLO BK evolution. This work is sponsored in part by the U.S. Department of Energy under Grant No. DE-SC0004286.

\section{REFERENCES}

1. Y. V. Kovchegov, Phys. Lett. B710, 192-196 (2012), 1112 . 2598.

2. Y. V. Kovchegov, and E. Levin, Nucl. Phys. B577, 221-239 (2000), hep-ph/9911523.

3. I. Balitsky, Nucl. Phys. B463, 99-160 (1996), hep-ph/9509348.

4. Y. V. Kovchegov, Phys. Rev. D60, 034008 (1999), hep-ph/9901281.

5. Y. Hatta, E. Iancu, C. Marquet, G. Soyez, and D. N. Triantafyllopoulos, Nucl. Phys. A773, 95-155 (2006), hep-ph/0601150.

6. Y. Kovchegov, and H. Weigert, Nucl. Phys. A 784, 188-226 (2007), hep-ph/ 0609090.

7. I. I. Balitsky, Phys. Rev. D 75, 014001 (2007), hep-ph / 0609105.

8. Y. Hatta, E. Iancu, K. Itakura, and L. McLerran, Nucl. Phys. A760, 172-207 (2005), hep-ph/ 0501171.

9. I. Balitsky, and G. A. Chirilli, Phys. Rev. D77, 014019 (2008), 0710.4330.

10. Y. Y. Balitsky, and L. N. Lipatov, Sov. J. Nucl. Phys. 28, 822 (1978).

11. E. A. Kuraev, L. N. Lipatov, and V. S. Fadin, Sov. Phys. JETP 45, 199-204 (1977).

12. Y. V. Kovchegov, L. Szymanowski, and S. Wallon, Phys. Lett. B586, 267-281 (2004), dedicated to the memory of Jan Kwiecinski, hep-ph/0309281.

13. I. Balitsky, and G. A. Chirilli, Nucl. Phys. B822, 45-87 (2009), 0903.5326.

14. I. Balitsky, and G. A. Chirilli (2012), 1207 . 3844.

15. A. Sabio Vera, Nucl.Phys. B746, 1-14 (2006), hep-ph/ 0602250.

16. J. Bartels, L. Lipatov, and G. Vacca, Phys. Lett. B477, 178-186 (2000), hep-ph/9912423.

17. M. A. Braun, Eur. Phys. J. C 53, 59 (2008), 0707.2314.

18. R. C. Brower, M. Djuric, and C.-I. Tan, JHEP 0907, 063 (2009), 0812 . 0354.

\footnotetext{
${ }^{2}$ Note that Mikhail Braun showed that running-coupling corrections do not change the LO odderon intercept, leaving it at zero [17]: our result (10) is consistent with this conclusion.
} 